\title{
The magnetic resonance enterography imaging features of symptomatic Meckel's diverticulum in pediatric patients: a retrospective observational study of 31 cases
}

\author{
Jun Hu, Chuan-Gao Yin, Ke-Fei Hu, Geng-Wu Li \\ Department of Radiology, Anhui Provincial Children's Hospital, Children's Hospital of Fudan University Anhui Hospital, Hefei, China \\ Contributions: (I) Conception and design: J Hu; (II) Administrative support: CG Yin; (III) Provision of study materials or patients: KF Hu; (IV) \\ Collection and assembly of data: J Hu, CG Yin; (V) Data analysis and interpretation: KF Hu, GW Li; (VI) Manuscript writing: All authors; (VII) \\ Final approval of manuscript: All authors. \\ Correspondence to: Chuan-Gao Yin. Department of Radiology, Anhui Provincial Children's Hospital, No. 39 Wangjiang East Road, Baohe District, \\ Hefei 230051, China. Email: ycgyindr@163.com.
}

\begin{abstract}
Background: The aim of this study was to explore the magnetic resonance enterography (MRE) imaging manifestations of a symptomatic Meckel's diverticulum (MD) in pediatric patients in order to provide a reference for the diagnosis of the condition.

Methods: The medical records of 31 pediatric patients with MD from May 2014 to October 2020 were retrospectively analyzed. The inclusion criteria were patients with MD accompanied by unexplained gastrointestinal bleeding, anemia (except hematological diseases), chronic persistent abdominal pain, repeated intussusception, or intussusception in older pediatric patients during surgery. The clinical variables (age, sex, and hemoglobin) and imaging, surgical, and pathological findings were recorded.

Results: MD was definitively identified in 28 patients, with the following characteristics: a blind-ending fluid-filled and/or gas-filled structure $(n=23)$, an elongated shape $(n=1)$, a dumbbell shape $(n=1)$, and a solid mass $(n=3)$. The diverticula were located in the right lower quadrant $(n=16)$, the right abdomen at the level of the umbilicus $(n=3)$, the right upper quadrant $(n=2)$, the left upper quadrant $(n=2)$, and the midline lower abdomen $(n=5)$. Supply arteries were visualized in nine cases. In all cases, mural enhancement was comparable to that of the adjacent small-bowel (SB). Extravasation of the intravascular contrast medium was seen in two cases. Peripheral structural abnormalities included mesenteric fat stranding $(n=7)$, hemorrhage in the adjacent lumen $(n=3)$, free intraperitoneal gas $(n=1)$, abnormal fluid retention $(n=2)$, intestinal obstruction $(\mathrm{n}=1)$, and lymph node enlargement $(\mathrm{n}=7)$. A normal appendix was identified in 18 cases.

Conclusions: MRE is an appropriate method of diagnosing symptomatic MD in pediatric patients and is particularly useful in the assessment of complications.
\end{abstract}

Keywords: Meckel's diverticulum (MD); diverticulum; magnetic resonance imaging (MRI); gastrointestinal hemorrhage; intestinal obstruction

Submitted Dec 03, 2020. Accepted for publication May 25, 2021.

doi: $10.21037 / \mathrm{tp}-20-419$

View this article at: https://dx.doi.org/10.21037/tp-20-419

\section{Introduction}

A Meckel's diverticulum (MD) is a congenital digestive tract malformation that is caused by the failure of the vitelline duct to obliterate (1). The incidence rate is about $1-3 \%$, and the ratio of male to female is about 3:1 (2). Most people with MD are asymptomatic for life; however, the condition often leads to complications, including intestinal hemorrhage, vomiting and distension resulting from an obstruction or perforation, and abdominal pain (3). It has been reported that the majority of patients with MD are 
younger than 24 months of age (4).

The development of unconsciousness and nonspecific symptoms may make the diagnosis of MD difficult. In particular, patients with unexplained shock should be suspected of acute abdomen before any other evidence is sought. The clinical presentation and results of physical examination and laboratory tests are also typically nonspecific; therefore, the preoperative diagnosis of $\mathrm{MD}$ in children is often challenging. Recently, pediatric magnetic resonance enterography (MRE) has been found to be useful in evaluating a variety of non-inflammatory bowel disease (non-IBD) conditions affecting the bowel and mesentery $(1,5)$. MRE can be used to evaluate the intestinal cavity and wall, the adjacent mesentery and soft tissue, and any extraintestinal abdominal renal-pelvis IBD manifestations. Therefore, the present study aimed to review the MRE features of MD in pediatric patients and proposed that MRE can be used as an auxiliary method of diagnosis accompanying radionuclide and ultrasound (US) imaging.

We present the following article in accordance with the STROBE reporting checklist (available at http://dx.doi. org/10.21037/tp-20-419).

\section{Methods}

\section{Subjects}

The medical records of 31 pediatric patients with symptomatic MD who had undergone MRE and, subsequently, a surgical resection with curative intent at our institution between May 2014 and October 2020 were retrospectively analyzed.

The study was conducted in accordance with the Declaration of Helsinki (as revised in 2013). The study was approved by the Ethics Committee of Anhui Provincial Children's Hospital and individual consent for this retrospective analysis was waived.

Inclusion criteria: (I) participants: (i) a pathologic diagnosis of MD in the small intestine was made; (ii) clinical symptoms of unexplained gastrointestinal bleeding, anemia (except hematological diseases), chronic persistent abdominal pain, repeated intussusception, or intussusception in older children [secondary intussusception caused by pathological lead point (PLP) was suspected]; (II) intervention: (i) based on the related clinical symptoms and MRE, patients with suspected MD were treated with surgery; (III) comparators: (i) MRE protocol sequence parameters; (ii) electronic medical records of 31 pediatric patients with pathologically proven MD; (iii) MRE characteristics of 31 pediatric patients with pathologically proven MD; (IV) outcomes: (i) all cases recovered and were discharged from hospital with good prognosis; (ii) related complications disappeared; (V) study design: (i) retrospective reports.

\section{Collection of demographic and clinical data}

The relevant electronic medical records were reviewed to document the following: (I) patient demographic data (including age and sex); (II) clinical presentation; (III) duration of symptoms; (IV) clinical indications for MRE; (V) other diagnostic imaging modalities; (VI) operative and pathological results. Preoperative findings obtained using other modalities, including ${ }^{99 \mathrm{~m}} \mathrm{Tc}$ scintigraphy, B-mode US, and computed tomography (CT), were also reviewed.

\section{Diagnostic criteria of MD}

Four criteria were used for the diagnosis of MD.

(I) MD was defined as a blind-ending outpouch of the bowel arising from the distal ileum. The distal ileum was typically found by detecting the cecum and terminal ileum and following the ileum in a retrograde fashion proximally from the terminal ileum. When such a structure was identified, MD was considered to be visible.

(II) The wall of the structure was thicker than that of the adjacent intestine, with limited diffusion and significant enhancement, suggesting diverticulitis.

(III) Adjacent fat-layer swelling, inflammatory exudation, and reactive lymph node enlargement, suggesting diverticulitis.

(IV) Short T1 signal or extravasation of the contrast agent after enhancement in the lumen, indicating diverticulum bleeding. A thin-layer balanced turbo-field echo (BTFE) sequence showed that the supplying artery was a branch of the ileocolic artery of the superior mesenteric artery.

\section{Pediatric MRE protocol}

MR images were acquired using a 1.5-T MR system (Achieva 1.5-T; Philips Healthcare, The Netherlands) with a 16-channel phased array coil. Before and after intravenous 
Table 1 MRE protocol sequence parameters used in this study

\begin{tabular}{|c|c|c|c|c|c|c|}
\hline Parameter & SS-TSE & $\begin{array}{c}\text { Fat-suppressed- } \\
\text { SS-TSE }\end{array}$ & $\begin{array}{c}\text { Two-dimensional } \\
\text { BTFE }\end{array}$ & $\begin{array}{c}\mathrm{DWI}^{\star} \text { (echo planar } \\
\text { single shot) }\end{array}$ & $\begin{array}{l}\text { T1-weighted turbo } \\
\text { field echo** }\end{array}$ & THRIVE \\
\hline Plane of view & Coronal, axial & Axial & Coronal & Coronal, axial & Coronal, axial & Coronal, axial \\
\hline Fat suppression & No & SPAIR & No & No & No & Yes \\
\hline Section thickness (mm) & 6.0 & 6.0 & 6.0 & 6.0 & 6.0 & 3.0 \\
\hline Repetition time (ms) & 1300 & 1300 & Shortest & Shortest & 10 & Shortest \\
\hline Echo time (ms) & 120 & 120 & Shortest & Shortest & 4.6 & Shortest \\
\hline
\end{tabular}

The shortest repetition time and/or echo time was set to be the shortest allowed by the imager. ${ }^{*}, \mathrm{~b}$ values of 0 , and 800 sec/mm ${ }^{2}$; ${ }^{*}$, this sequence was used for patients who unable to hold their breath. MRE, magnetic resonance enterography; SS-TSE, single-shot turbo spin-echo; BTFE, balanced turbo-field echo; DWI, diffusion-weighted imaging; THRIVE, T1-weighted high-resolution isotropic volume examination; SPAIR, spectral attenuated inversion recovery.

administration of a contrast medium (Magnevist, Bayer Pharma AG, Germany) at a dose of $0.1 \mathrm{mmol} / \mathrm{kg}$, images with the following sequences were obtained: (I) a respiratory-triggered (RT) coronal and axial T2-weighted single-shot turbo spin-echo (SS-TSE) sequence; (II) an RT axial T2-weighted SS-TSE sequence with fat saturation; (III) an RT coronal two-dimensional BTFE sequence; (IV) RT coronal and axial diffusion-weighted images with $\mathrm{b}$ values of 0 and $800 \mathrm{~s} / \mathrm{mm}^{2}$; (V) a coronal and axial T1-weighted $3 \mathrm{D}$-gradient recalled echo with fat saturation [T1-weighted high-resolution isotropic volume examination (THRIVE), Philips Healthcare, The Netherlands]. The images were acquired during the arterial- and portal-venous phases. Multisequence and multiplanar imaging were performed as detailed in Table 1.

\section{Preparation of pediatric patients without sedation}

MRE examinations were performed after 4 hours of fasting. During the 45-minute period prior to imaging, an oral biphasic contrast material with mannitol $2.5 \%$ was administered (dosage $15 \mathrm{~mL} / \mathrm{kg}$ ). To reduce small-bowel (SB) peristalsis and prolong SB distension, scopolamine $0.3 \mathrm{mg} / \mathrm{kg}$ (Shanghai Xinyi Pharmaceuticals Co., Ltd., China) was intravenously administered 10 minutes before the examination.

\section{Preparation of pediatric patients with sedation}

A total of $20(20 / 31,64.5 \%)$ MRE examinations were performed under sedation (deep sedation in spontaneously breathing patients); chloral hydrate was orally administrated at a dose of $0.5 \mathrm{~mL} / \mathrm{kg}$, in accordance with hospital protocol. The SB was filled by manual injection through a fluoroscopically placed nasojejunal tube. After the imaging was complete, residual gastric contents were suctioned via a gastric drainage tube prior to extubation to minimize the risk of aspiration. The patients were monitored as they gradually awakened from sedation.

\section{Qualitative and quantitative analyses}

The MR images were independently reviewed by two abdominal radiologists, with 14 and 24 years of abdominal magnetic resonance imaging (MRI) interpretation experience, respectively, using a picture archiving and communication system (PACS; RISGC 3.0.4.1, Carestream Health, KODAK). Both observers were aware of the MD diagnosis.

Eight imaging parameters were evaluated for the qualitative and quantitative analyses: (I) visualization: $\mathrm{MD}$ was defined as a blind-ending outpouch of the bowel arising from the distal ileum; when such a structure was identified 
on the MRE, MD was considered to be visible; (II) location: divided into four quadrants by the midline and at the level of the umbilicus; (III) size: diameter was measured on the axial images; (IV) mural: thickness, enhancement, and/or edema compared with the intestinal wall; (V) dominant contents of the lumen: fluid, gas, or hemorrhagic material (defined as high-SI foci on T1-weighted images, with variable SI on T2-weighted images; (VI) the presence or absence of active contrast extravasation, supply arteries, and gastric mucosa signs (markedly thickened mucosa similar to gastric mucosa); (VII) peripheral structural abnormalities: mesenteric fat infiltration, hyperemia or stranding, hemorrhage in the adjacent lumen, free intraperitoneal gas, abnormal fluid retention, intestinal obstruction, and lymph node enlargement (defined as $\geq 1.5 \mathrm{~cm}$ diameter on axial images); (VIII) identification of a normal appendix.

\section{Statistical analysis}

Statistical analysis was performed using SPSS 13.0 (SPSS Inc., Chicago, IL, USA). The normality of variables was analyzed using a Shapiro-Wilk test. Quantitative variables were represented as mean \pm standard deviation $(\mathrm{SD})$, and discrete variables were represented as frequencies or percentages.

\section{Results}

\section{Subject population}

The mean age $(\bar{x} \pm$ SD) of pediatric patients with symptomatic MD was $3.8 \pm 3.3$ years (range, 8 months to 12 years). The male to female ratio was approximately $4: 1(81 \%$ vs. $19 \%)$. The presentations were unexplained gastrointestinal bleeding $(\mathrm{n}=23,74.2 \%)$, abdominal pain $(\mathrm{n}=9,29.0 \%)$, intussusception $(\mathrm{n}=5,16.1 \%), \mathrm{SB}$ obstruction (SBO) $(n=1,3.2 \%)$, fever $(n=2,6.5 \%)$, vomiting $(n=2,6.5 \%)$, and diarrhea $(\mathrm{n}=1,3.2 \%)$. Laboratory testing revealed a mean hemoglobin level of $8.4 \pm 2.4$ (range, $5.1-13.7$ ) g/dL. The results of other imaging modalities for MD detection are summarized in Table 2.

MRE was performed successfully on 28 children; oral contrast material was not administered in three patients with intestinal obstruction to avoid disease aggravation. Minor adverse events because of contrast oral ingestion (abdominal discomfort, vomiting) were seen in 5 patients $(17.9 \%)$. No major adverse events were observed.

\section{Surgical and pathologic findings}

Thirty-one symptomatic MD cases were pathologically confirmed. The mean distance from the MD to the ileocecal valve was $41.6 \pm 24.3$ (range, $15-140$ ) $\mathrm{cm}$. The length and width of the diverticulum were $36.4 \pm 25.6$ (range, $15-110$ ) $\mathrm{mm}$ and $12.7 \pm 6.8$ (range, 5-40) mm, respectively. Ectopic gastric mucosa was seen in 28 patients (Figure 1), and pancreatic tissue was detected in three of them. The other pathologic findings are summarized in Table 2.

\section{MRE image review}

In the retrospective review, $\mathrm{MD}$ was definitively identified in 28 of the 31 patients, while the remaining three were undetectable. The appearance of the diverticula in the 28 identified MD cases was as follows: a blind-ending, fluid-filled and/or gas-filled structure $(\mathrm{n}=23)$ (Figure 1), an elongated shape $(\mathrm{n}=1)$ (Figure 2), a dumbbell shape $(\mathrm{n}=1)$ (Figure 3), and a solid mass $(\mathrm{n}=3)$ (Figure 4). The MD was most commonly located in the right abdomen either in the right upper quadrant $(\mathrm{n}=2)$ (Figure 3), right lower quadrant $(\mathrm{n}=16)$ (Figure 2), or right abdomen at the level of the umbilicus $(\mathrm{n}=3)$ (Figure 1). In a small number of patients, the MD was located in the left abdomen or midline abdomen, either in the left upper quadrant $(n=2)$ or the midline lower abdomen $(\mathrm{n}=5)$. The mural thickness of the diverticula ranged from 2 to 7.2 (mean, $4.1 \pm 1.2$ ) $\mathrm{mm}$. The walls of the diverticula showed mural enhancement comparable with that of the adjacent SB in all the patients. Mild inflammatory changes were present in the adjacent mesentery in seven patients with visualized diverticula (Figure 4). Supply arteries were observed in nine cases. Small mesenteric lymph nodes measuring $>1.5 \mathrm{~cm}$ in the short-axis dimension were visualized in seven patients (Figure 1). Active contrast extravasation was visualized in two (Figure 5), while a normal appendix was visualized in 18 patients (Figure 6). The other MRI manifestations are summarized in Table 3 and Figures 1-6.

\section{Discussion}

$\mathrm{MD}$ is a congenital intestinal blind pouch that results from an incomplete obliteration of the vitelline duct during the fifth week of gestation. Most cases of MD are found within $100 \mathrm{~cm}$ of the ileocecal valve. In the present study, the mean distance to the ileocecal valve was $41.36 \pm 24.3 \mathrm{~cm}$. A MD is lined with heterotopic mucosa in up to $60 \%$ cases, with 


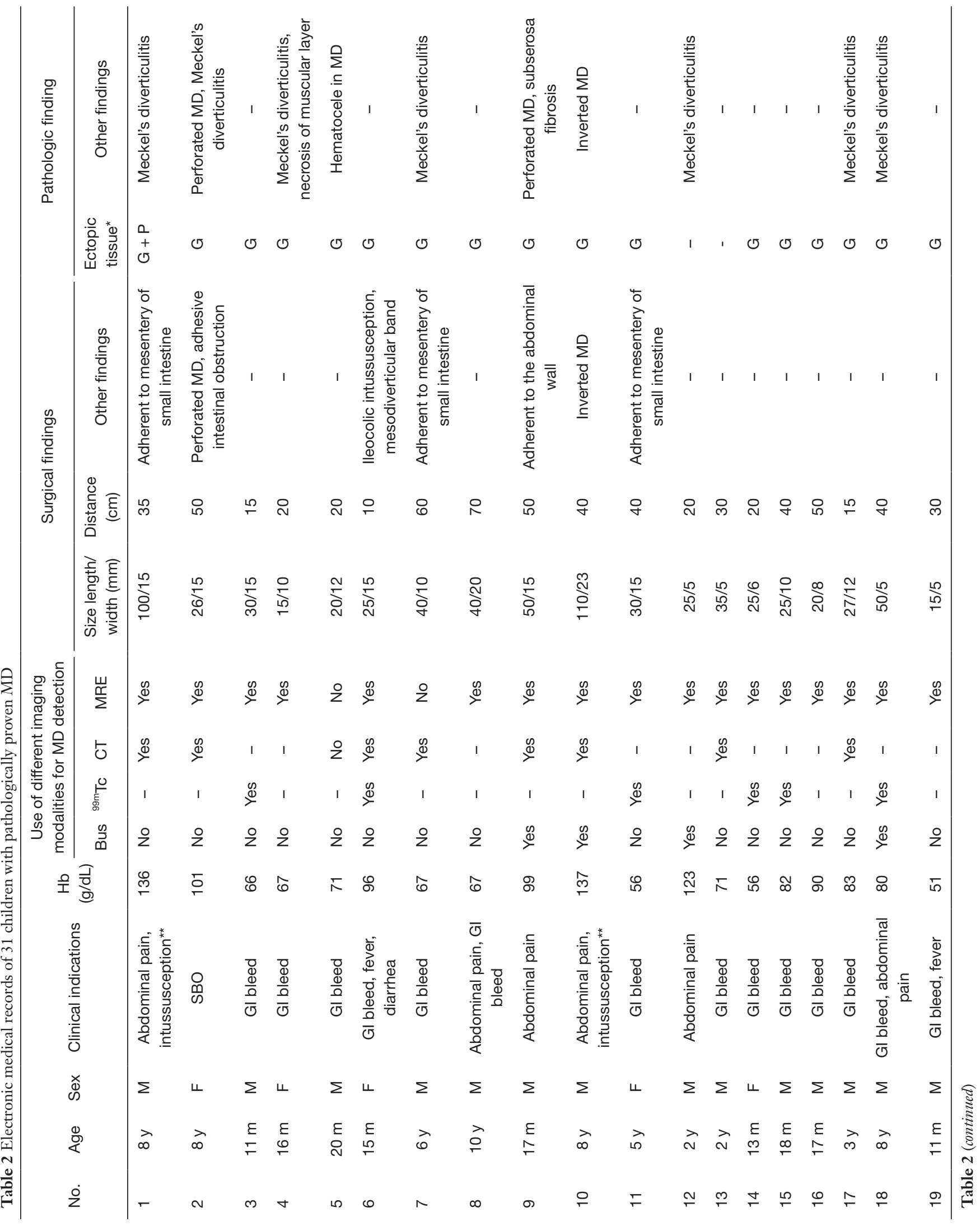




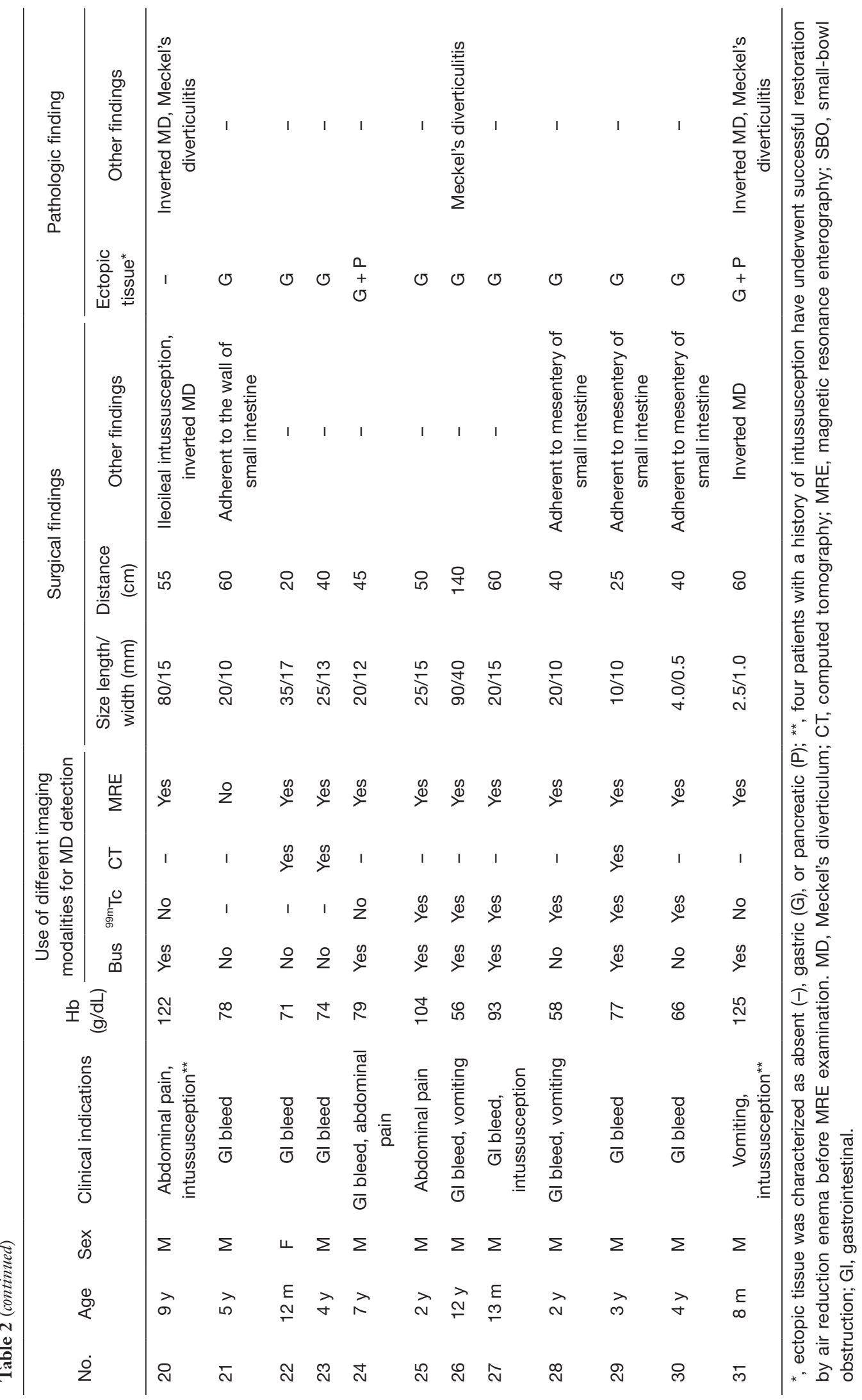



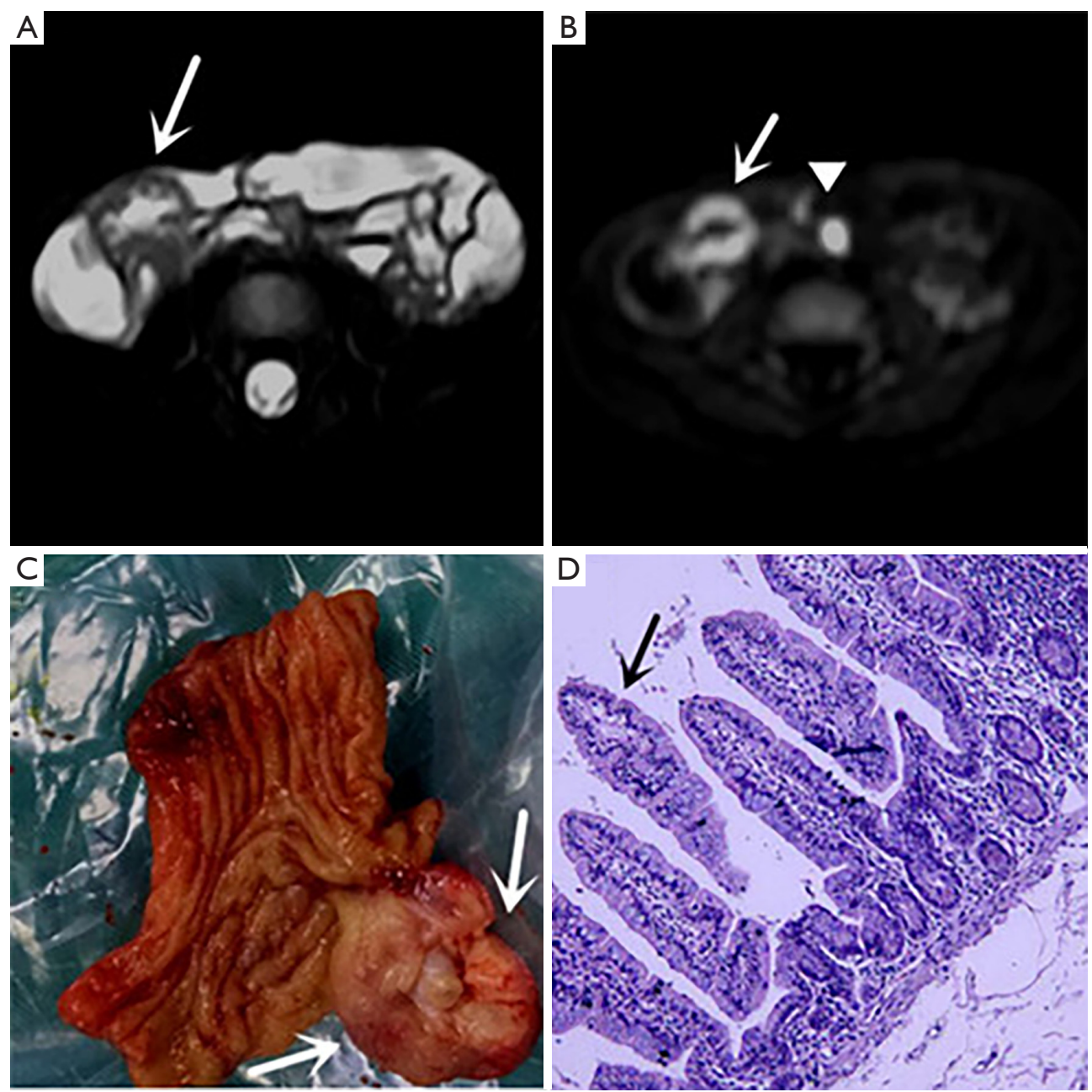

Figure $1 \mathrm{MRE}$ in an 18-month-old boy, who presented with lower gastrointestinal hemorrhage (case 15 in Tables 2,3). The axial fatsuppressed SS-TSE image (A) shows a blind-ending, fluid-filled outpouch (arrow) in the right abdomen at the level of the umbilicus, and the marked thickened mucosa is similar to gastric mucosa (gastric mucosa sign). The axial DWI (b=800) image (B) shows a restricted diffusion wall (arrow), and adjacent reactive mesenteric lymph nodes appearing as a restricted diffusion (arrowhead). The surgically resected specimen image (C) and the microscopic image (D) (hematoxylin and eosin stain, 200x) confirm the presence of heterotopic gastric epithelium (arrow) within the MD (arrows). MRE, magnetic resonance enterography; SS-TSE, single-shot turbo spin-echo; DWI, diffusion-weighted imaging; MD, Meckel's diverticulum.

the following distribution: gastric mucosa, $62 \%$; pancreatic, $6 \%$; both gastric and pancreatic, $5 \%$; jejunal, $2 \%$; Brunner's glands, 2\%; gastric and duodenal, $2 \%(6-8)$. This is consistent with the case etiology noted in the present study.

The existence of MD can cause a number of complications in pediatric patients, including hemorrhage, intestinal obstruction, inflammation, and perforation $(9,10)$, most of which require surgical intervention. Multiple imaging modalities (11) aid in the diagnosis of symptomatic MD. Abdominal scintigraphy with ${ }^{99 \mathrm{~m}} \mathrm{Tc}$-pertechnetate is a well-established diagnostic technique used in the evaluation of pediatric patients with lower gastrointestinal tract bleeding, enabling the detection of heterotopic gastric mucosa in MD (12). The sensitivity of this imaging technique is approximately $85 \%(13,14)$. However, a MD without ectopic gastric mucosa will not be visible on a ${ }^{99 \mathrm{~m}}$ Tc-pertechnetate scan. Furthermore, scintigraphy results can be falsely positive owing to enteric duplication cysts containing heterotopic gastric mucosa, intussusception, heterotopic gastric mucosa on otherwise normal intestines, 


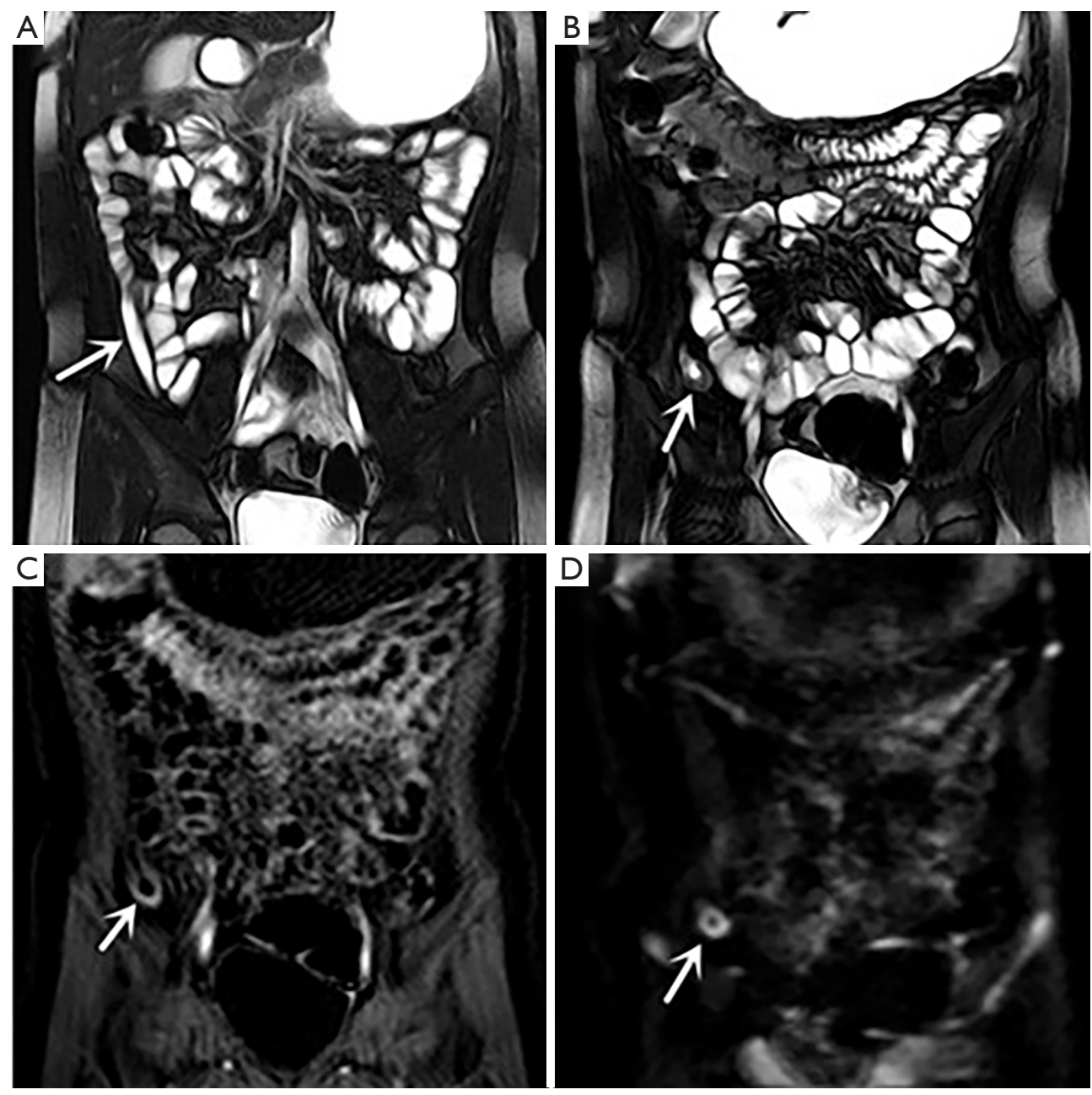

Figure $2 \mathrm{MRE}$ in an 8-year-old boy, who presented with lower gastrointestinal hemorrhage and abdominal pain (case 18 in Tables 2,3). The coronal BTFE images (A) and (B) show an elongated, blind-ending, fluid-filled structure (arrow) in the right lower quadrant, with a marked thickened wall (arrow) at the tip of the MD. The coronal contrast-enhanced THRIVE image (C) shows a marked hyper-enhancement wall (arrow). The coronal DWI ( $b=800)$ image (D) shows a restricted diffusion wall (arrow). As a normal appendix was not identified, this structure was preoperatively misdiagnosed as appendicitis. A surgical and pathological diagnosis of MD was established, and the appendix was found to be normal. MRE, magnetic resonance enterography; BTFE, balanced turbo-field echo; MD, Meckel's diverticulum; THRIVE, T1-weighted high-resolution isotropic volume examination; DWI, diffusion-weighted imaging.

or intestinal inflammatory processes $(15,16)$.

As the preferred primary imaging modality in the evaluation of pediatric patients, US has several advantages, including the absence of ionizing radiation and the need for sedation, easy availability, and low cost. However, the disadvantages of US are its dependence on operator skill, its limited evaluation of alternative diagnoses and complications, and its low negative predictive value. The observed US-based MD visualization rate in the present study was only $54.5 \%(18 / 33)$.
CT, another important imaging modality used for the diagnosis of a symptomatic MD (17-20), can clearly depict a variety of complications. However, the use of CT in pediatric patients has been questioned and moderated because of concerns over the potential negative effects of ionizing radiation (21). Unfortunately, radiologic data regarding the utility of MRE for MD assessment is scarce $(5,22)$.

The present study shows how MRE can be used to visualize the diverticulum's location, shape, mural, contents, 

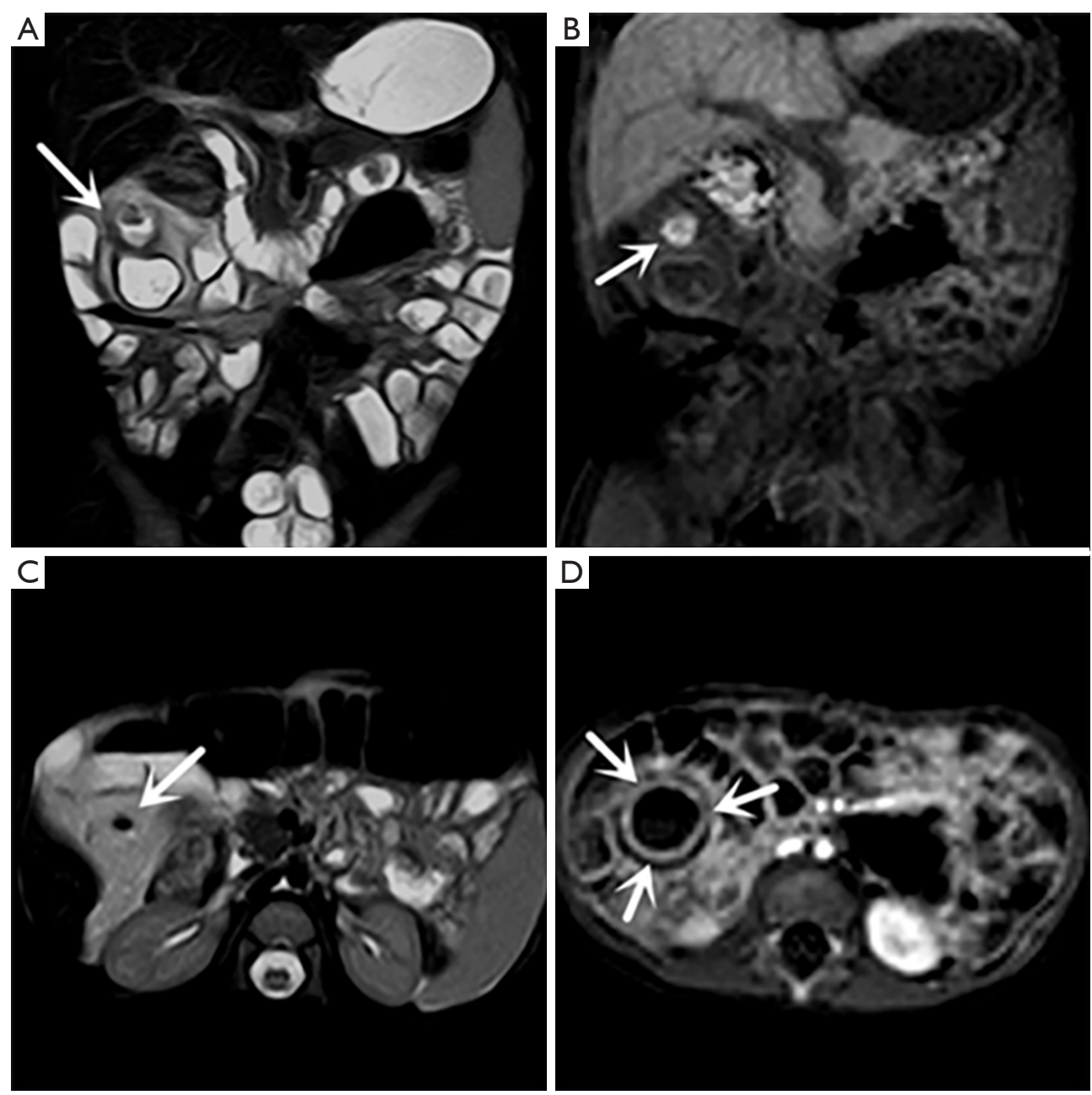

Figure $3 \mathrm{MRE}$ in a 17-month-old boy, with a pathologically proven MD with a perforation, who presented with a history of intermittent abdominal pain (case 9 in Tables 2,3). The coronal SS-TSE image (A) demonstrates a fluid-filled outpouch with a dumbbell shape (arrow) in the right upper quadrant surrounded by inflamed fat. The coronal THRIVE image (B), confirming that the MD content is hemorrhagic, shows a high-intensity signal (arrow). The axial SS-TSE image (C) shows free intraperitoneal gas (arrow) in the inflammatory mesenteric fat. The axial contrast-enhanced THRIVE image (D) demonstrates a marked hyper-enhancement wall (arrows). MRE, magnetic resonance enterography; MD, Meckel's diverticulum; SS-TSE, single-shot turbo spin-echo; THRIVE, T1-weighted high-resolution isotropic volume examination.

and peripheral structural abnormalities. The observed MD visualization rate of MRE in the present study was $90.3 \%$ (28/31). Even though the reviewers were not blinded to the surgical and pathological results, the diverticulum was undetected in three of the cases and a suggestive diagnosis was made in one case based on the sign of the intravascular contrast medium. In accordance with the operative and pathological results, this lack of detection could be due to several factors, including a small, collapsed diverticulum that exceeds the spatial resolution of MRI, a MD with minor complications (which is often mistaken for a normal SB loop) $(3,15,16,18)$, and breathing artifacts.

In 21 of the 28 identified cases (75\%), the diverticulum was most commonly located in the right abdomen (15). The cases with MD located in the right lower quadrant therefore needed to be differentiated from appendicitis, for which the identification of a normal appendix was key. Bennett et al. (23) reported that administration of oral contrast material is invaluable in enabling appendix identification, and previous studies have reported appendix 

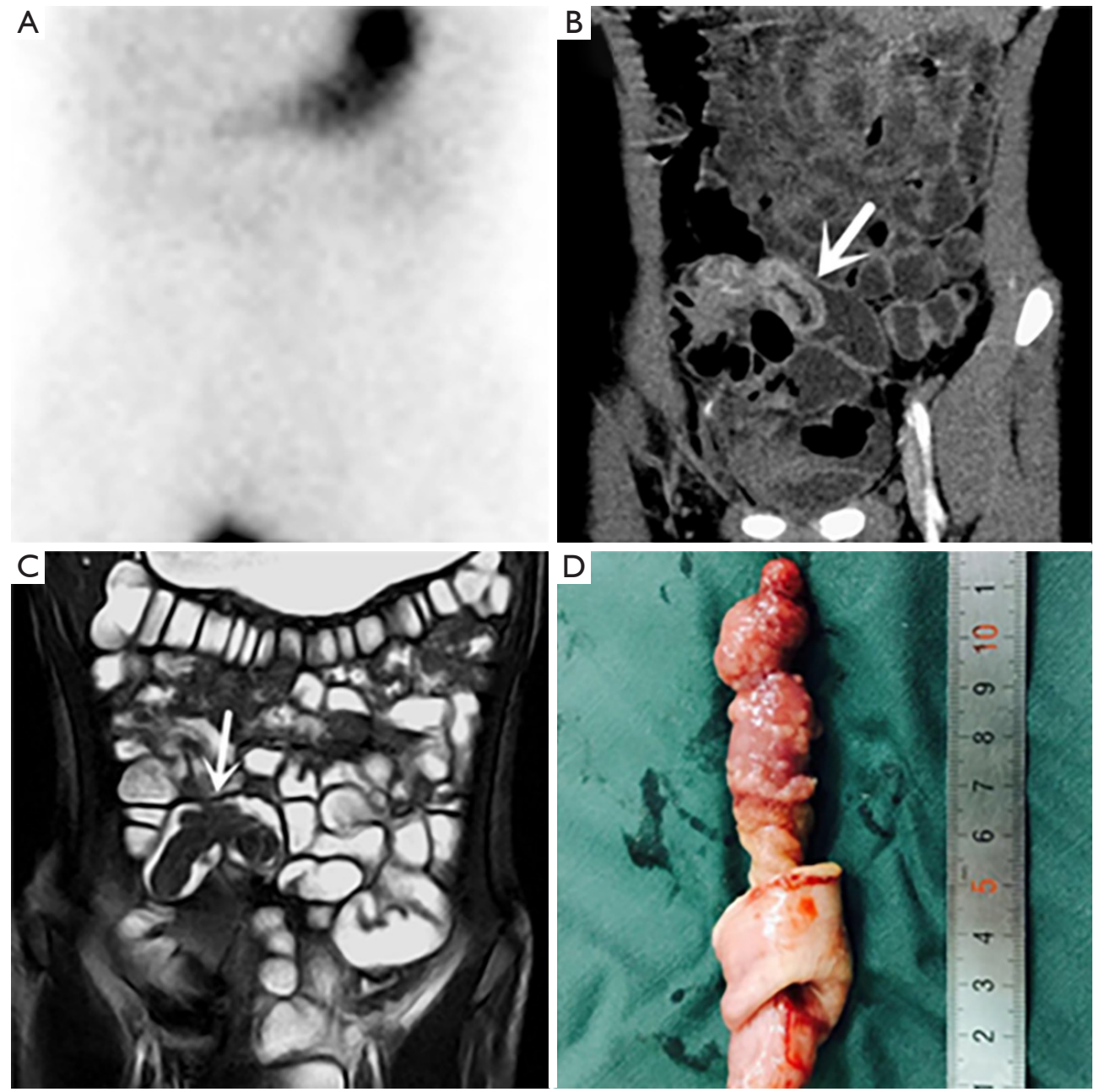

Figure 4 An 8-year-old boy, who presented with intermittent abdominal pain and who has a history of intussusception (case 10 in Tables 2,3). The ${ }^{99 \mathrm{~m}} \mathrm{Tc}$ pertechnetate study in image (A) is negative. The coronal contrast-enhanced CT image (B) demonstrates an elongated intraluminal fat-attenuating lesion (arrow) in the pelvic ileal loop. The coronal BTFE image (C) shows an elongated, polypoid intraluminal mass (arrows) with smooth margins running parallel to the long axis of the bowel loop, which corresponds to the lesion shown in (B). The surgically resected specimen image (D) shows a 11-cm-long, elongated intraluminal mass in the proximal ileum, which was proven to be an inverted MD. CT, computed tomography; BTFE, balanced turbo-field echo; MD, Meckel's diverticulum.

visualization rates of $74.8-86.8 \%$ with MRI $(24,25)$. However, the observed appendix visualization rate in the present study $(18 / 31,58.1 \%)$ was lower than in prior reports, despite most of the patients ingesting the contrast material.

The MRE images showed that the diverticulum mostly appeared as a blind-ending, tubular, fluid-filled or gas-filled structure (23/28). In three cases, however, an inverted MD appeared as a polypoid intraluminal mass, in one case it appeared as an elongated shape in the right lower quadrant and was misdiagnosed as appendicitis in the initial official report, and in another case it appeared as a dumbbell shape. In nine cases, the markedly thickened mucosa showed a gastric mucosal shape that was defined as the gastric mucosal sign. All cases were pathologically confirmed by the presence of heterotopic gastric epithelium. This suggests that the gastric mucosal sign may be a special indicator of MD with heterotopic gastric epithelium. Nonetheless, further studies with larger samples are needed to validate these findings.

In line with the signal characteristics of combinatorial sequences, the contents of the diverticulum, such as 

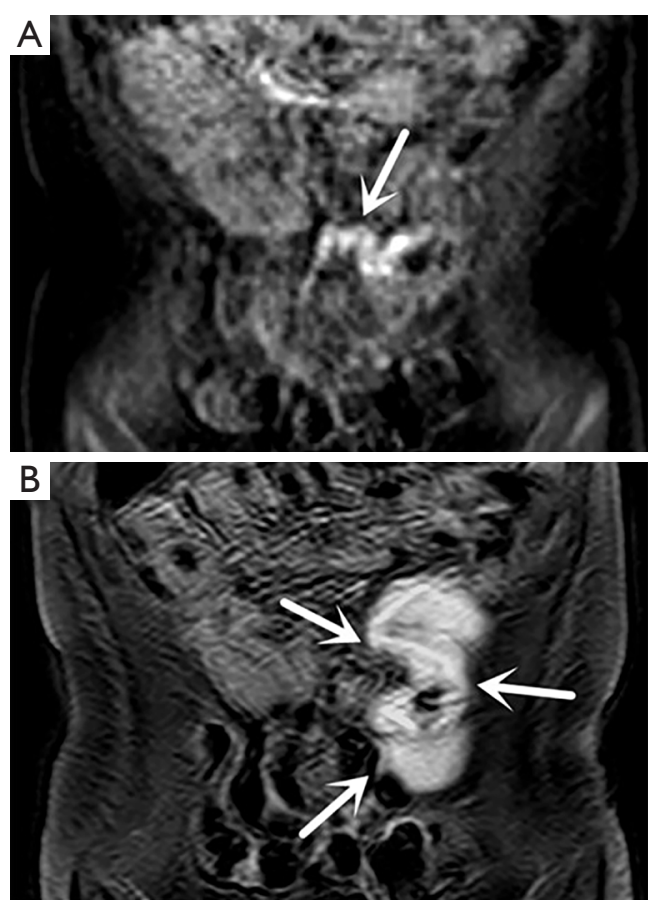

Figure 5 An MRE of a 6-year-old boy, with a pathologically proven bleeding MD, who presented with lower gastrointestinal hemorrhage (case 7 in Tables 2,3). Coronal gadolinium-enhanced THRIVE image (A) at arterial phase demonstrates contrast medium extravasation due to active bleeding (arrow), and delayed enhanced THRIVE image (B) shows large amounts of contrast accumulated in the adjacent lumen (arrows). MRE, magnetic resonance enterography; MD, Meckel's diverticulum; THRIVE, T1-weighted high-resolution isotropic volume examination.

hemorrhagic material, gas, and the ingested contrast agent, can be read in MRE images. MD appears as a blind-ending, tubular, thick-walled structure with wall enhancement and soft-tissue stranding of the adjacent mesenteric fat. In the present study, this structure appeared markedly hyperintense on diffusion-weighted imaging (DWI), suggesting the presence of restricted diffusion, which allowed for considerably good detection close to the wall enhancement. MRE may very rarely show extravasation of intravascular contrast medium in the case of active bleeding in MD. This may be due to three factors: most cases of active bleeding are controlled before the MRE examination; as with CT, active bleeding may be detected when the bleeding exceeds $0.3-0.5 \mathrm{~mL} / \mathrm{min}$ (20); the temporal resolution is limited. However, when active bleeding is present at the time of scanning, luminal extravasation of contrast material may occur. This feature was seen only in

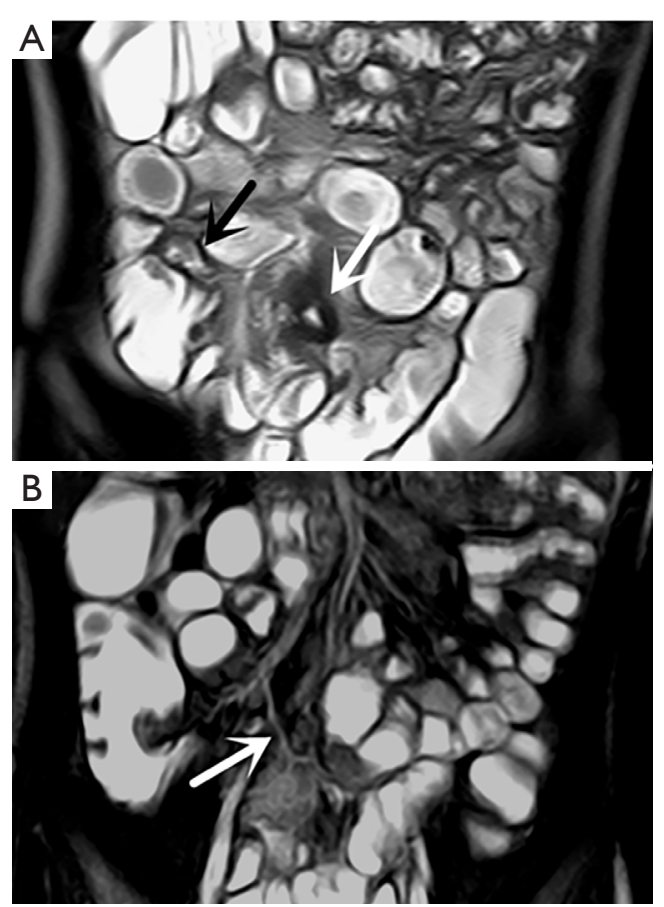

Figure $6 \mathrm{MRE}$ in an 8-year-old boy with pathologically proven MD, who presented with abdominal pain and a history of intussusception (case 1 in Tables 2,3). Coronal SS-TSE image (A) demonstrates a tubular, blind-ending, fluid-filled outpouch with markedly thickened wall (white arrow) in the right lower quadrant; a normal appendix (black arrow) is seen separate from the MD. Coronal BTFE image (B) shows one of the branches of the superior mesenteric artery, consistent with MD (arrow). MRE, magnetic resonance enterography; MD, Meckel's diverticulum; SS-TSE, single-shot turbo spin-echo; BTFE, balanced turbo-field echo.

three cases in the present study.

The BTFE sequence is a fully balanced, steady-state, coherent imaging pulse sequence that uses a very short repetition time and is designed to provide high signal levels within a short time. BTFE is therefore a robust imaging modality to simultaneously visualize both the bowel and blood vessels. One of the branches (the persistent vitellointestinal artery) of the superior mesenteric artery, consistent with MD, may be visualized on BTFE images, and it accounted for approximately $32.1 \%(9 / 28)$ of the cases in the present study. This suggests that the visualization of supply arteries might ensure a more confident diagnosis of MD.

Past studies have reported an inverted MD (26-28). With $\mathrm{CT}$, an inverted $\mathrm{MD}$ appears as an intraluminal fatty mass 


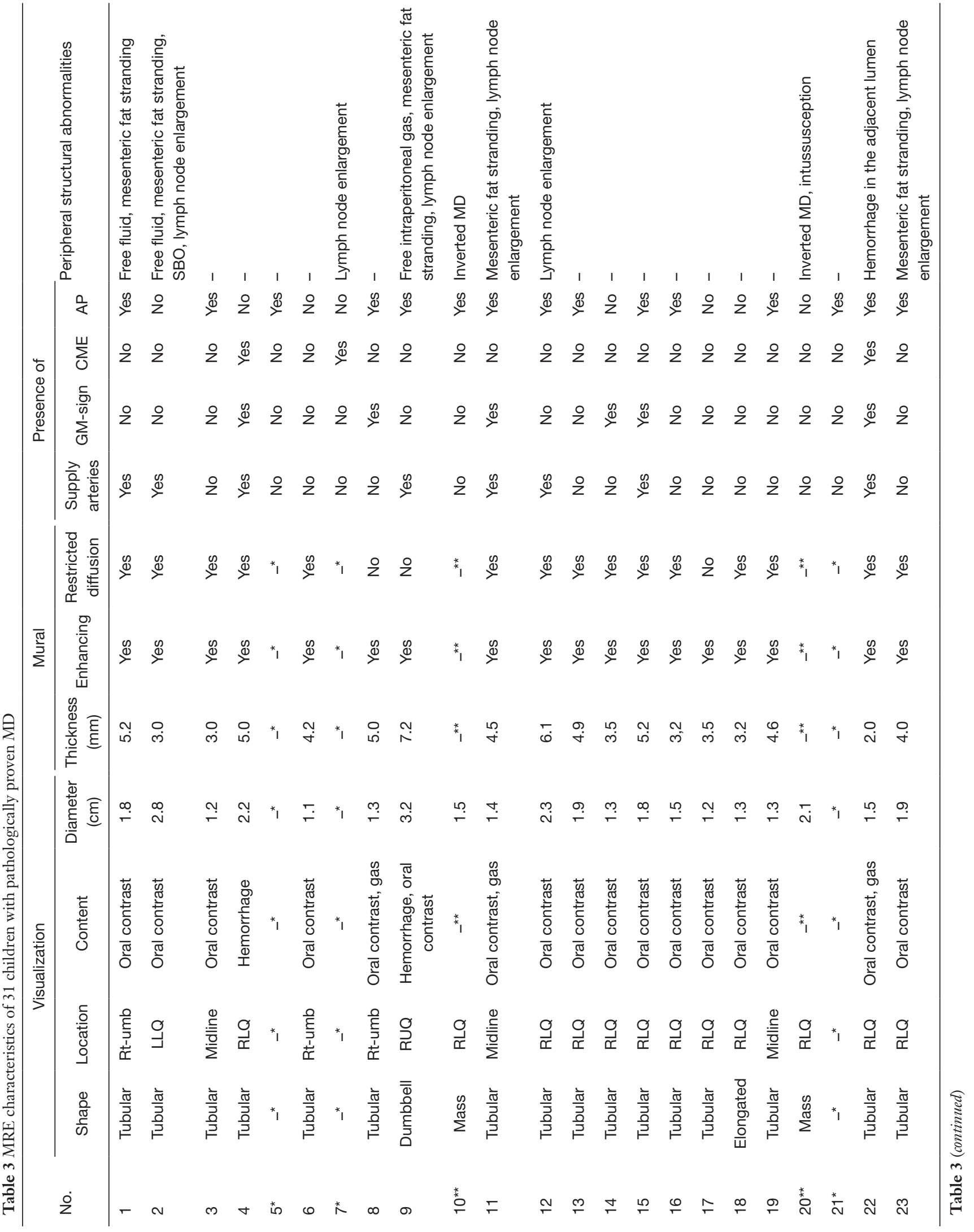




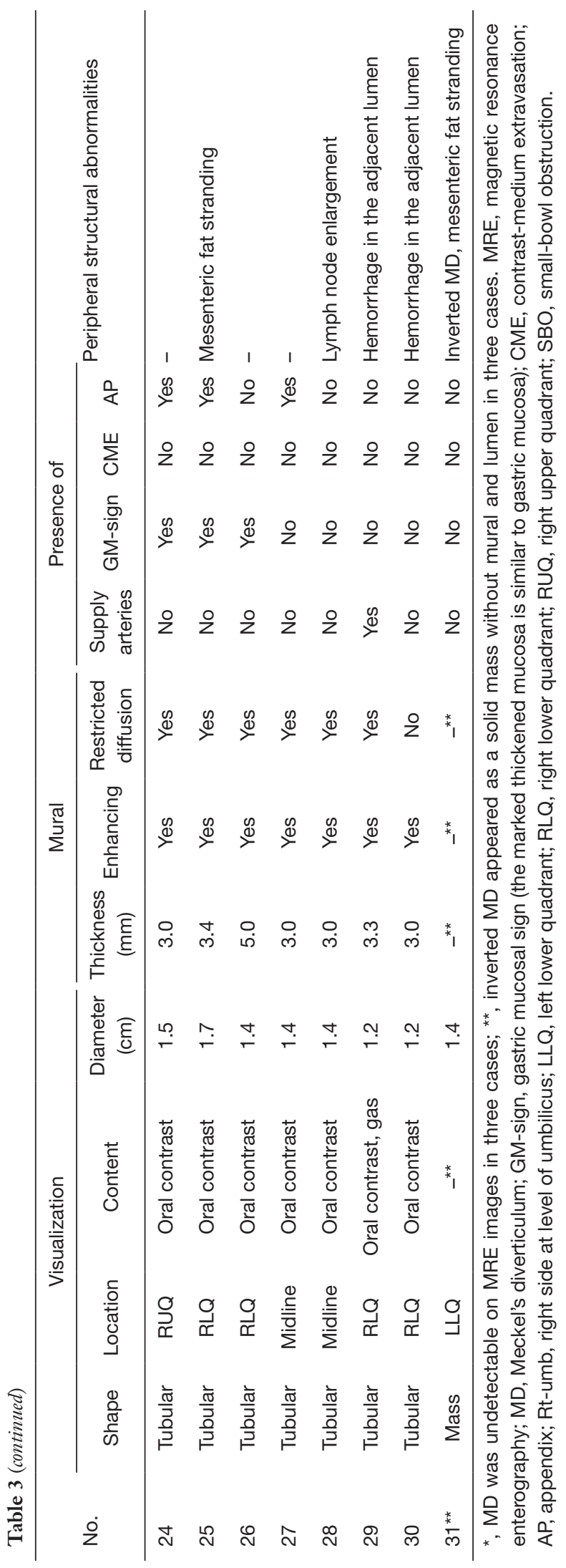

that corresponds to the entrapped perienteric fatty tissue within the inverted serosal side of the diverticulum (28); if associated with intussusception, it may also have a characteristic coiled spring appearance. As MRE is a combination of enterographic and tomographic images, it effectively presents the inverted diverticulum as an elongated mass with smooth margins and a mobile intraluminal polypoid mass, which ran parallel to the long axis of the bowel in the ileum in three cases in the present study. A perforated MD can be predicted by the presence of free intraperitoneal air on CT $(11,18)$; this feature was also seen in the MRE images in the present study.

The present study has some limitations. First, it was a single-center, retrospective study with a small number of cases. Second, the reviewers were aware that all the MRE studies were of patients with surgically proven MD, and they were not blinded, which could have caused reviewer bias: a visualized MD could have been confused with other conditions, such as appendicitis, intestinal duplication cysts, or IBD $(6,29,30)$. Furthermore, two radiologists reviewed the images in consensus, so quantifiable detectability (i.e., interobserver agreement quantified with a kappa value) could not be calculated. Third, the study did not include a negative control group, so it was not possible to evaluate false positives in the detection of MD. Last, 20 patients were given the oral contrast agent through a fluoroscopically placed nasojejunal tube during sedation. Although no major adverse events occurred in the present study, and Sadigh et al. $(31,32)$ reported that fluid instillation via nasojejunal intubation with a patient under general anesthesia is a safe technique, the risk of aspiration cannot be ignored. Accordingly, further research is needed to determine whether conventional abdominal MRI is a safer alternative for the diagnosis of MD in younger children.

\section{Conclusions}

Although preliminary, the present study indicates that MRE could be a valid alternative in the diagnosis of MD, allowing for an accurate diagnosis of location and the characterization of complications while at the same time avoiding exposure to radiation. Using multiple sequences in MRE can also provide more evidence for the diagnosis of MD and its complications. More specifically, MRE can facilitate the visualization of the diverticulitis and its contents, supply arteries, gastric mucosal sign, and peripheral structural abnormalities. Although there is not yet sufficient evidence for MRE to be routinely used as 
the primary imaging modality, the findings of the present study suggest that it could be used in an emergency in cases of clinically suspected MD, especially where US or scintigraphy results are suspected to be false negatives.

\section{Acknowledgments}

Funding: This work was supported by the Anhui Provincial Health Commission (No. 2019SEY005).

\section{Footnote}

Reporting Checklist: The authors have completed the STROBE reporting checklist. Available at http://dx.doi. org/10.21037/tp-20-419

Data Sharing Statement: Available at http://dx.doi. org/10.21037/tp-20-419

Conflicts of Interest: All authors have completed the ICMJE uniform disclosure form (available at http://dx.doi. org/10.21037/tp-20-419). The authors have no conflicts of interest to declare.

Ethical Statement: The authors are accountable for all aspects of the work in ensuring that questions related to the accuracy or integrity of any part of the work are appropriately investigated and resolved. The study was conducted in accordance with the Declaration of Helsinki (as revised in 2013). The study was approved by the Ethics Committee of Anhui Provincial Children's Hospital and individual consent for this retrospective analysis was waived.

Open Access Statement: This is an Open Access article distributed in accordance with the Creative Commons Attribution-NonCommercial-NoDerivs 4.0 International License (CC BY-NC-ND 4.0), which permits the noncommercial replication and distribution of the article with the strict proviso that no changes or edits are made and the original work is properly cited (including links to both the formal publication through the relevant DOI and the license). See: https://creativecommons.org/licenses/by-nc-nd/4.0/.

\section{References}

1. Mollard BJ, Smith EA, Dillman JR. Pediatric MR enterography: technique and approach to interpretationhow we do it. Radiology 2015;274:29-43.
2. Vaabengaard S, Andersen L, Qvist N, et al. Complicated Meckel's diverticulum in children: clinical presentation, diagnostic work-out, surgical approach and postoperative complications. Cureus 2020;12:e12354.

3. Platon A, Gervaz P, Becker CD, et al. Computed tomography of complicated Meckel's diverticulum in adults: a pictorial review. Insights Imaging 2010;1:53-61.

4. Malhotra S, Roth DA, Gouge TH, et al. Gangrene of Meckel's diverticulum secondary to axial torsion: a rare complication. Am J Gastroenterol 1998;93:1373-5.

5. Casciani E, Nardo GD, Chin S, et al. MR Enterography in paediatric patients with obscure gastrointestinal bleeding. Eur J Radiol 2017;93:209-16.

6. Hegde S, Dillman JR, Gadepalli S, et al. MR enterography of perforated acute Meckel diverticulitis. Pediatr Radiol 2012;42:257-62.

7. Epifanio M, Terrazas E, Spolidoro J, et al. Magnetic resonance findings of a Meckel diverticulum at $3 \mathrm{~T}$. $\mathrm{J}$ Pediatr Gastroenterol Nutr 2017;64:e13.

8. Matsagas MI, Fatouros M, Koulouras B, et al. Incidence, complications, and management of Meckel's diverticulum. Arch Surg 1995;130:143-6.

9. Menezes M, Tareen F, Saeed A, et al. Symptomatic Meckel's diverticulum in children: a 16-year review. Pediatr Surg Int 2008;24:575-7.

10. Kusumoto H, Yoshida M, Takahashi I, et al. Complications and diagnosis of Meckel's diverticulum in 776 patients. Am J Surg 1992;164:382-3.

11. Kotha VK, Khandelwal A, Saboo SS, et al. Radiologist's perspective for the Meckel's diverticulum and its complications. Br J Radiol 2014;87:20130743.

12. Sfakianakis GN, Haase GM. Abdominal scintigraphy for ectopic gastric mucosa: a retrospective analysis of 143 studies. AJR Am J Roentgenol 1982;138:7-12.

13. Ymaguchi M, Takeuchi S, Awazu S. Meckel's diverticulum. Investigation of 600 patients in Japanese literature. Am J Surg 1978;136:247-9.

14. Sfakianakis GN, Conway JJ. Detection of ectopic gastric mucosa in Meckel's diverticulum and in other aberrations by scintigraphy: I. Pathophysiology and 10-year clinical experience. J Nucl Med 1981;22:647-54.

15. Swaniker F, Soldes O, Hirschl RB. The utility of technetium $99 \mathrm{~m}$ pertechnetate scintigraphy in the evaluation of patients with Meckel's diverticulum. J Pediatr Surg 1999;34:760-4; discussion 765.

16. Kotecha M, Bellah R, Pena AH, et al. Multimodality imaging manifestations of the Meckel diverticulum in children. Pediatr Radiol 2012;42:95-103. 
17. Kawamoto S, Raman SP, Blackford A, et al. CT detection of symptomatic and asymptomatic Meckel diverticulum. AJR Am J Roentgenol 2015;205:281-91.

18. Olson DE, Kim YW, Donnelly LF. CT findings in children with Meckel diverticulum. Pediatr Radiol 2009;39:659-63; quiz 766-7.

19. Won Y, Lee HW, Ku YM, et al. Multidetector-row computed tomography (MDCT) features of small bowel obstruction (SBO) caused by Meckel's diverticulum. Diagn Interv Imaging 2016;97:227-32.

20. Artigas JM, Martí M, Soto JA, et al. Multidetector CT angiography for acute gastrointestinal bleeding: technique and findings. Radiographics 2013;33:1453-70.

21. Mathews JD, Forsythe AV, Brady Z, et al. Cancer risk in 680,000 people exposed to computed tomography scans in childhood or adolescence: data linkage study of 11 million Australians. BMJ 2013;346:f2360.

22. Zhou FR, Huang LY, Xie HZ. Meckel's diverticulum bleeding diagnosed with magnetic resonance enterography: a case report. World J Gastroenterol 2013;19:2727-30.

23. Bennett GL, Birnbaum BA, Balthazar EJ. CT of Meckel's diverticulitis in 11 patients. AJR Am J Roentgenol 2004;182:625-9.

24. Koning JL, Naheedy JH, Kruk PG. Diagnostic performance of contrast-enhanced MR for acute appendicitis and alternative causes of abdominal pain in

Cite this article as: $\mathrm{Hu} \mathrm{J}$, Yin CG, Hu KF, Li GW. The magnetic resonance enterography imaging features of symptomatic Meckel's diverticulum in pediatric patients: a retrospective observational study of 31 cases. Transl Pediatr 2021;10(8):1974-1988. doi: 10.21037/tp-20-419 children. Pediatr Radiol 2014;44:948-55.

25. Mushtaq R, Desoky SM, Morello F, et al. First-line diagnostic evaluation with MRI of children suspected of having acute appendicitis. Radiology 2019;291:170-7.

26. Pantongrag-Brown L, Levine MS, Elsayed AM, et al. Inverted Meckel diverticulum: clinical, radiologic, and pathologic findings. Radiology 1996;199:693-6.

27. Kim JH, Park SH, Ha HK. Case 156: inverted Meckel diverticulum. Radiology 2010;255:303-6.

28. Kim KH, Kang KA, Lim JH, et al. Inverted Meckel diverticulum as a lead point of small bowel intussusception: misinterpreting case as a lipoma. Clin Imaging 2016;40:840-2.

29. Levy AD, Hobbs CM. From the archives of the AFIP. Meckel diverticulum: radiologic features with pathologic Correlation. Radiographics 2004;24:565-87.

30. Kim YS, Kim DJ, Bang SU, et al. Intestinal duplication cyst misdiagnosed as Meckel's diverticulum. Chin Med J (Engl) 2016;129:235-6.

31. Sadigh S, Chopra M, Sury MR, et al. Paediatric magnetic resonance enteroclysis under general anaesthesia - initial experience. Pediatr Radiol 2017;47:877-83.

32. Mollard BJ, Smith EA, Lai ME, et al. MR enterography under the age of 10 years: a single institutional experience. Pediatr Radiol 2016;46:43-9. 